\title{
INSTABILITIES IN A POLYTROPIC ATMOSPHERE
}

\author{
H.M. Antia and S.M. Chitre \\ Tata Institute of Fundamental Research \\ Bombay, India
}

Abstract

The density in the outer layers of stars varies by several orders of magnitude and it is desirable to include the full effects of compressibility in any study of instabilities arising in stellar convection zones. In an unstable compressible fluidlayer that is thermally conducting both the oscillatory and non-oscillatory motions can simultaneously arise. The conditions under which the oscillatory acoustic modes can be overstabilized in a polytropic atmosphere are examined. It is argued that the linearized perturbation theory breaks down when applied to an inviscid complete polytrope which has vanishing density and temperature at the top for both optically-thin and optically-thick approximations. However, the linearized theory is demonstrated to be self-consistent when viscosity and thermal conductivity are included in the study of complete polytropes.

\section{ON THE DYNAMICS OF THE SOLAR CONVECTION ZONE}

Bernard R. Durney and Hendrik C. Spruit

National Center for Atmospheric Research

Boulder, CO 80303 U.S.A.

Abstract

We derive expressions for the turbulent viscosity and turbulent conductivity applicable to convection zones of rotating stars. We assume that the dimensions of the convective cells are known and derive a simple distribution function for the turbulent convective velocities under the influence of rotation. From this distribution function (which includes, in particular, the stabilizing effect of rotation on convection) we calculate in the mixing-length approximation: i) the turbulent Reynolds stress 
tensor and $i i)$ the expression for the heat flux in terms of the superadiabatic gradient. The contributions of the turbulent convective motions to the mean momentum and energy equation are treated consistently, and assunptions about the turbulent viscosity and heat transport are replaced by assumptions about the turbulent flow itself. The free parameters in our formalism are the relative cell sizes and their dependence on depth and latitude.

\section{THERMAL AND CONTINUUM DRIVEN CONVECTION IN B-STARS}

George Driver Nelson

CODE CB, Johnson Space Center

Houston, Texas, 77058

Abstract

Two regions of convective instability are present in the photosphere of a typical B-star $\left(\mathrm{T}_{\text {eff }}=30,000 \mathrm{~K} \log \mathrm{g}=4.0\right)$. One is the usual thermal instability caused by the helium ionization. The other is driven by the continuum radiation pressure in a thermally stable layer. Mixing length and anelastic modal representations of these unstable regions show that the rapid radiative cooling of temperature fluctuations limits the velocities to an amplitude of a few meters per second, much too small to account for the observed line broadening and asymetries. 